\title{
Study of Indoor Positioning Method Based on Combination of Support Vector Regression and Kalman Filtering
}

\author{
Yu Zhang ${ }^{1,2}$, Lian Dong ${ }^{1}$, Lei Lai ${ }^{1}$ and Lizhi $\mathrm{Hu}^{1}$ \\ 1. Shanghai Institute of Measurement and Testing Technology, Shanghai, China; \\ 2. East China Normal University, Shanghai, China \\ \{zhang_yu,dongl, lail,hulz\}@simt.com.cn
}

\begin{abstract}
Against the problem that indoor positioning suffers quite large errors and irregular user location movement, this paper adopts Support Vector Regression (SVR) for initial positioning and Kalman filtering for filtering of the positioning results so as to improve the accuracy of the positioning system. The experimental results show that against the real WLAN environment, SVR positioning results processed by Kalman filtering indicates the root mean square error is decreased by $16 \%$, and $73 \%$ of positioning accuracy within 2 meters is increased to $83 \%$.
\end{abstract}

Keywords: Indoor positioning; SVR; Kalman filtering; positioning accuracy

\section{Introduction}

With the rapid development of mobile communication technology, Location Based Services (LBS), a location-based and service-oriented technology came into being and quickly gained widespread attention of the community, which reflects the importance of its core technology - positioning [1][2]. So far, the outdoor positioning technology has been relatively mature, focusing on the global positioning system, such as GPS, GLONASS and Beidou satellite navigation and positioning systems to achieve all-weather real-time positioning. In terms of study and utilization of outdoor GPS positioning technology, research on the indoor positioning technology started late, and the current indoor positioning focuses on positioned based on WLAN signal strength, utilizing the correlation of the existing indoor Received Signal Strength (RSS) signal and position information to achieve positioning[3-6]. Currently, RSS-based indoor positioning algorithms mostly adopt the position fingerprint technology [7-10]. Different from the positioning technology based the signal time of arrival (TOA) and angle of arrival (AOA) $[11,12]$, fingerprint positioning does not require any additional hardware to develop precise time synchronization or angular measurements. It can take advantage of existing wireless network infrastructure, greatly reducing the cost of the system and achieving a wide range of applicability. Location fingerprinting algorithms can be divided into two phases: Offline and online phases. In the offline phase, it is necessary to select a number of reference points and collect RSS at each reference point position corresponding to multiple Access Point (AP), establishing database storing each reference point location and fingerprint corresponding to RSS. In the online phase, it is necessary to compare RSS obtained in the terminal real time measurement with RSS in the fingerprint data, estimating the position of the terminal. Traditional online matching algorithms include $\mathrm{K}$ Nearest Neighbor (KNN) [13], Support Vector Machine (SVM) [14] and probability distribution [15].

In case of real-time follow-up applications, the system shall calculate the user's current location within a short time to achieve real-time, resulting in less samples of the signal strength obtained by the terminal. In addition, affected by uncertainties in the surrounding environment, the signal may witness greater fluctuation in a short time. These two factors 
will reduce the positioning accuracy of the system. Therefore, calculating the user's position directly with the conventional positioning algorithm makes user's location characterized by a large variance in the user's location and irregular user's mobile position and significant jump, seriously affecting the indoor positioning system's performance and stability. To solve the above problem, this paper proposes the SK algorithm combining SVR and Kalman Filtering, firstly applying SVR algorithm to obtain the location coordinates to be determined, then applying Kalman Filtering to filter the user's location coordinates estimated by positioning algorithm, making the user's location movement regular to improve the positioning accuracy of the indoor positioning system. Simulation and real experimental results show that the filtered position is significantly improved.

\section{Related Work}

Many position technologies and applications have been proposed. Microsoft research first proposed the indoor position system RADAR based on WLAN, which takes the RSS as character parameter of position and uses the K-nearest neighbor (KNN) to realize position match [16]. In open indoor environment, the system can obtain accuracies of 2-3 meters, using 70 access points that are placed non-uniformly at least 2.5 meters, but accuracy is not ideal in complex indoor environment.

In 2002, Horus system models the probability statistic, and stores the RSS Gaussian distribution in radio map [17]. At the same time, the block cluster concept first is proposed. The system decreases computational complex and improves positioning accuracy compared to the others.

Ekahau is a real-time position system that localizes the position of terminal based on statistic conditional probability by comparing the different between the received signal RSS and radio map [18]. The positioning accuracy of the system can achieve 1meter.

With the application of new algorithms and new theory in WLAN indoor position area, many advanced WLAN position systems come up. For example, a position method is proposed based on manifold regularization by J.J. Pan, et. al. [19]. The system that utilizing the RSS sample both of labeled position coordinate and unlabeled position coordinate is different from conditional WLAN indoor position system. The method is a two-step procedure. The first step, which is generally carried out offline, is the collection and process part labeled RSS sample, then computes the figure Laplace matrix. Finally, an estimation function of position coordinate is obtained by training using the above matrix and manifold learning theory. The second step firstly preprocesses unlabeled online RSS data, and position result is obtained through putting the processed date into estimation function.

\section{SVR Location Algorithm}

Based on the structural risk minimization theory, the support vector machine (SVM) learning machine comprehensively considers learning function $\mathrm{VC}$ dimension and training errors, seeking the learning functions minimizing the actual risks so as to improve the generalization capacity of the learning machine. It has multiple unique advantages in nonlinear and high dimensional pattern recognitions. SVM can be divided into support vector classification (SVC) and support vector regression (SVR). SVR function regression has been successfully applied to the system identification and nonlinear system prediction.

\subsection{Overview}

This paper SVR positioning includes offline and online phases:

(1) Offline phase: 
In the offline phase, it is necessary to utilize a mobile device to collect original training data sets consisting of RSS information from each AP at the known reference points of the pre-selected position coordinates. Considering the indoor environment is usually complex, witnessing different factors significantly impacting RSS signal distribution such as wall reflections and people walking, it is necessary to filter the original training data sets so as to achieve higher reliability of the data sets and properly characterize RSS distribution against the indoor wireless environment. After filtration, the data sets are taken as input conditions and SVR is utilized for supervised learning so as to achieve the corresponding position prediction model.

(2) On-line phase:

In the on-line phase, it is necessary to utilize a mobile device to collect ambient wireless signals at the position points of the unknown coordinates and obtain a RSS data set. It is necessary to filter the data set by the means similar to that of the offline phase. The position prediction model made in the offline phase and the RSS data sets obtained in the online phase are utilize to predict obtained RSS data sets location prediction and obtain the position coordinates of the indoor mobile targets.

\subsection{Location Prediction Model}

Position prediction model describes the physical location of the mobile device and the relationship between it and RSS information received from adjacent wireless access points. Suppose there are $\mathrm{n}$ AP and $\mathrm{N}$ reference points in the positioning system, RSS information received by the mobile device at Position A is $s_{A}=\left\{r s s_{A}^{1}, r s s_{A}^{2}, \cdots, r s s_{A}^{n}\right\}, r s s_{A}^{i}$ is RSS of No. $\mathrm{i}$ wireless access point received by the mobile device at Position A, is the position coordinates of the mobile device at Position A, the given data set $D:=\left\{\left(s_{i}, L_{i}\right)\right\}_{i=1}^{N}\left(s_{i} \in R, L_{i} \in L\right)$, the target of function regression theory is to seek mapping $f: R \rightarrow L$ : achieving $f\left(s_{i}\right) \approx L_{i}$. The mapping relationship belongs to non-linear mapping relationships. Based on the support vector machine method, in terms of nonlinear, it is proper to utilize nonlinear function $\Phi(s)$ to project the input space $R$ into the high dimensional feature space $R^{\prime}$, and utilize the linear function to fit the data $\operatorname{set}\left\{\left(\Phi\left(s_{i}\right), L_{i}\right)\right\}_{i=1}^{N}$ in the high dimensional feature space:

$$
L=f(s)=W^{T} \Phi(s)+b
$$

Where $W \in R$ is weight vector, $b$ is bias term.

According to the statistics theory, it is proper to calculate take the following objective function (training error function) minimum value and obtain $W, b$ :

$$
J(w)=\frac{1}{2}\|w\|^{2}+\lambda \sum_{i=1}^{N}\left|L_{i}-\left(W^{T} \Phi(s)+b\right)\right|_{\varepsilon}
$$

Where $\varepsilon, \lambda$ is the empirical parameter, $\left|L_{i}-\left(W^{T} \Phi(s)+b\right)\right|_{\varepsilon}$ is called $\varepsilon$ insensitive loss function, the value taking is as follows:

$$
\left|L_{i}-\left(W^{T} \Phi(s)+b\right)\right|_{\varepsilon}=\left\{\begin{array}{l}
0, \quad\left|L_{i}-\left(W^{T} \Phi(s)+b\right)\right|<\varepsilon \\
L_{i}-\left(W^{T} \Phi(s)+b\right)-\varepsilon, \text { else }
\end{array}\right.
$$

That is, when the error of the predicted value is less than $\varepsilon$, the loss function value is taken as 0 ; otherwise, the linear punishment shall be taken.

Introduce two slack variables $\xi_{i}, \xi_{i}^{\prime}$, equivalent to the following optimization, 
achieving:

$$
\begin{aligned}
& \min \frac{1}{2}\|w\|^{2}+C\left(\sum_{i=1}^{N}\left(\xi_{i}+\xi_{i}^{\prime}\right)\right) \\
& \text { s.t. }\left\{\begin{array}{l}
L_{i}-\left(W^{T} \Phi(s)+b\right) \leq \varepsilon+\xi_{i} \\
\left(W^{T} \Phi(s)+b-L_{i}\right) \leq \varepsilon+\xi_{i}^{\prime} \\
\xi_{i} \geq 0 \\
\xi_{i} \geq 0
\end{array}\right.
\end{aligned}
$$

Parameter $\varepsilon$ indicates the requirement of the system for regression function error on the training data set error, the smaller $\varepsilon$ is, the smaller the error of the regression function on the training data set will be and the higher the obtained regression function estimation accuracy will be and the more the support vectors will be. Parameter $\mathrm{C}$ is the punishment to the data set on the training data set with the regression estimated function error greater than $\varepsilon$, the greater $\mathrm{C}$ is, the greater the punishment to these data sets will be.

The above optimization can be defined as the following lagrangian function:

$$
\begin{array}{r}
L\left(w, \xi_{i}, \xi_{i}^{\prime}\right)=\frac{1}{2}\|w\|^{2}+C\left(\sum_{i=1}^{N}\left(\xi_{i}+\xi_{i}^{\prime}\right)\right)-\sum_{i=1}^{N}\left(\eta_{i} \xi_{i}+\eta_{i}^{\prime} \xi_{i}^{\prime}\right)- \\
\sum_{i=1}^{N} \alpha_{i}\left(W^{T} \Phi\left(s_{i}\right)-L_{i}+\varepsilon+\xi_{i}\right)-\sum_{i=1}^{N} \alpha_{i}^{\prime}\left(L_{i}-W^{T} \Phi\left(s_{i}\right)+\varepsilon+\xi_{i}\right)
\end{array}
$$

Where $\xi_{i}, \xi_{i}^{\prime}, \alpha, \alpha$ is lagrangian multiplier, when it acquires extremes, it is necessary to achieve

$$
\begin{gathered}
\frac{\partial L\left(w, \xi_{i}, \xi_{i}^{\prime}\right)}{\partial w}=w-\sum_{i=1}^{N}\left(\alpha_{i}-\alpha_{i}^{\prime}\right) \Phi\left(s_{i}\right)=0 \\
\frac{\partial L\left(w, \xi_{i}, \xi_{i}^{\prime}\right)}{\partial \xi_{i}}=\eta_{i}-\left(C-\alpha_{i}\right)=0 \\
\frac{\partial L\left(w, \xi_{i}, \xi_{i}^{\prime}\right)}{\partial \xi_{i}^{\prime}}=\eta_{i}^{\prime}-\left(C-\alpha_{i}^{\prime}\right)=0
\end{gathered}
$$

Make the above three formulas into the formula, and introduce kernel function $K\left(s_{i}, s_{j}\right)$ based on the SVR theory and utilize Wlofe dual techniques to transfer the above into the following corresponding dual optimization, achieving:

$$
\min \sum_{i=1}^{N} L_{i}\left(\alpha-\alpha_{i}^{\prime}\right)-\varepsilon \sum_{i=1}^{N}\left(\alpha_{i}-\alpha_{i}^{\prime}\right)-\frac{1}{2} \sum_{i=1}^{N} \sum_{j=1}^{N}\left(\alpha_{i}-\alpha_{i}^{\prime}\right)\left(\alpha_{j}-\alpha_{j}^{\prime}\right) K\left(s_{i}, s_{j}\right)
$$




$$
\text { s.t. }\left\{\begin{array}{l}
\sum_{i=1}^{N}\left(\alpha_{i}-\alpha_{i}^{\prime}\right)=0 \\
0 \leq \alpha_{i} \leq C \\
0 \leq \alpha_{i}^{\prime} \leq C
\end{array}\right.
$$

Thus, the corresponding regression formula can be changed to the following formula:

$$
L=\sum_{i=1}^{N}\left(\alpha_{i}-\alpha_{i}^{\prime}\right) K\left(s, s_{i}\right)+b
$$

This paper adopts RBF core $K\left(s_{i}, s_{j}\right)=\exp \left(-\frac{\left\|s_{i}-s_{j}\right\|^{2}}{2 \sigma^{2}}\right)$.

\section{Kalman Filtering Algorithm}

In real-time positioning system, it is necessary to calculate the user's position in a short period of time, which usually suffers small signal samples and large positioning errors. This paper proposes Kalman Filtering SVR positioning results SK algorithm so as to reduce SVR positioning errors and improve positioning accuracy.

Kalman Filtering is an effective Gauss process optimal filtering algorithm. When the object model is accurate enough and the system status and parameters are free from mutation, Kalman Filtering prediction equations and observation equations are:

$$
\begin{aligned}
& x_{k}=\Phi_{k, k-1} x_{k-1}+\Gamma_{k-1} \omega_{k-1} \\
& z_{k}=H_{k} x_{k}+m_{k} k \geq 1
\end{aligned}
$$

This paper assumes process noise and observation noise are Gauss white noise, satisfying:

$$
E\left\{w_{k}\right\}=0 \quad, \quad E\left\{m_{k}\right\}=0, \quad \operatorname{Cov}\left(w_{k}, w_{j}\right)=Q_{k} \delta_{k j}, \quad \operatorname{Cov}\left(m_{k}, m_{j}\right)=R_{k} \delta_{k j},
$$

$\operatorname{Cov}\left(w_{k} m_{j}\right)=0$,

$$
\delta_{k j}=\left\{\begin{array}{ll}
1 & k=j \\
0 & k \neq j
\end{array} .\right.
$$

Initial conditions:

$$
\begin{gathered}
\hat{x}_{0}=E\left[x_{0}\right]=\mu_{0} \\
\operatorname{Var} \tilde{x}_{0}=\operatorname{Varx}_{0}=P_{0}
\end{gathered}
$$

Kalman Filtering time update equations:

$$
\begin{gathered}
\hat{x}_{k / k-1}=\Phi_{k, k-1} \hat{x}_{k-1} \\
P_{k / k-1}=\Phi_{k, k-1} P_{k-1} \Phi_{k, k-1}^{\tau}+\Gamma_{k-1} Q_{k-1} \Gamma_{k-1}^{\tau}
\end{gathered}
$$

Measurement updates equations: 


$$
\left\{\begin{array}{l}
K_{k}=P_{k / k-1} H_{k}^{\tau}\left[H_{k} P_{k / k-1} H_{k}^{\tau}+R_{k}\right]^{-1} \\
\hat{x}_{k}=\hat{x}_{k / k-1}+K_{k}\left(z_{k}-H_{k} \hat{x}_{k / k-1}\right) \\
P_{k}=\left[I-K_{k} H_{k}\right] P_{k / k-1}
\end{array}\right.
$$

The 2-dimensional model of the user position is defined as follows:

$$
\begin{aligned}
& {\left[\begin{array}{c}
x_{k} \\
y_{k} \\
v_{k}^{x} \\
v_{k}^{y}
\end{array}\right]=\left[\begin{array}{llll}
1 & 0 & 1 & 0 \\
0 & 1 & 0 & 1 \\
0 & 0 & 1 & 0 \\
0 & 0 & 0 & 1
\end{array}\right]\left[\begin{array}{c}
x_{k-1} \\
y_{k-1} \\
v_{k-1}^{x} \\
v_{k-1}^{y}
\end{array}\right]+\left[\begin{array}{c}
w_{k}^{x} \\
w_{k}^{y} \\
w_{k}^{v x} \\
w_{k}^{v y}
\end{array}\right]} \\
& {\left[\begin{array}{c}
z_{k}^{x} \\
z_{k}^{y}
\end{array}\right]=\left[\begin{array}{llll}
1 & 0 & 0 & 0 \\
0 & 1 & 0 & 0
\end{array}\right]\left[\begin{array}{l}
x_{k} \\
y_{k} \\
v_{k}^{x} \\
v_{k}^{y}
\end{array}\right]+\left[\begin{array}{l}
m_{k}^{x} \\
m_{k}^{y}
\end{array}\right]}
\end{aligned}
$$

Where $x_{k}, y_{k}$ respectively represent moving target displacement in $\mathrm{X}$ direction and $\mathrm{Y}$ direction in the 2-dimensional plane, $v_{k}^{x}$ and $v_{k}^{y}$ correspond to the speed in $\mathrm{X}$ direction and $\mathrm{Y}$ direction.

\section{Experiment Results and Analysis}

Based on simulation scene and realistic scene, this paper compares properties of presented SK algorithm and KNN algorithm, SVR algorithm positioning accuracy root mean square error (RMSE), error percentage and cumulative distribution function (CDF) within 2 meters.

\subsection{Simulation Scene}

\subsubsection{Establishment of the Simulation Scene}

This paper first utilized the simulation environment to have a detailed performance analysis of the proposed positioning method. Positioning area was $40 \mathrm{~m} * 40 \mathrm{~m} .12$ Access Points (AP) were distributed around. In total 1600 reference points; spacing was $1 \mathrm{~m}$. Based on the path, 200 test points were selected. The simulation scene is shown in Figure 1. 


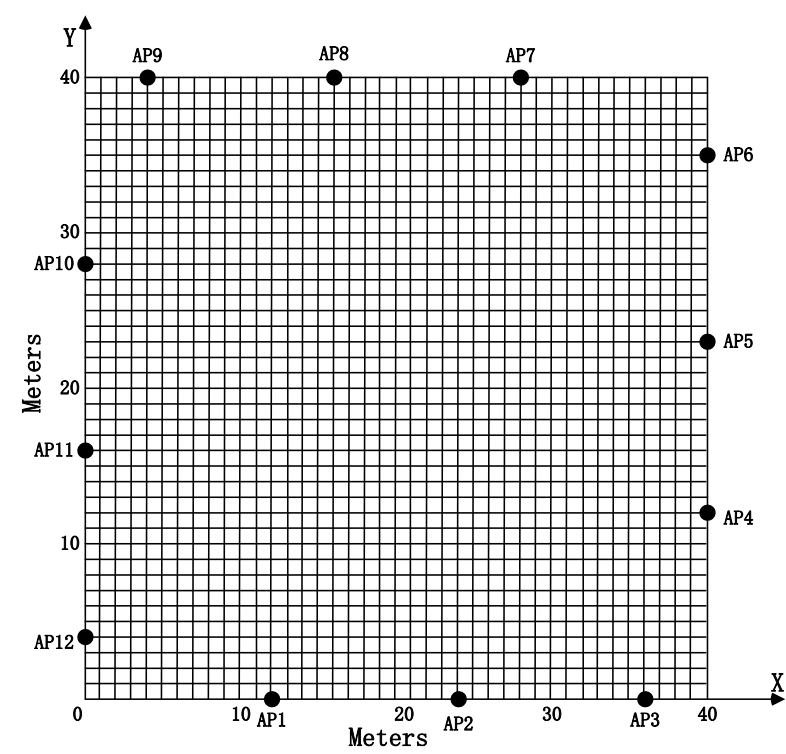

Figure 1. Simulation Scene

The following model was adopted to calculate average signal strength of each AP received at each sampling point position [20].

$$
\begin{array}{r}
p l\left(d_{A P_{i}, p}\right)= \\
R l\left(d_{0}\right)+10 \cdot \alpha \cdot \log _{10}(d)(17) \\
R S S_{A P_{i}, p}=P t-p l\left(d_{A P_{i}, p}\right)
\end{array}
$$

Where $d_{A P_{i}, p}$ represents the distance between access point $A P_{i}$ and sampling point $p$, $p l\left(d_{A P_{i}, p}\right)$ represents loss mean of $A P_{i}$ signal at Position $p$ from the access point, $p l\left(d_{0}\right)$ represents the path loss of signal transmission at reference distance $d_{0}=1 \mathrm{~m}$, the signal loss, $\alpha$ represents path loss coefficient, $P t$ represents transmit power of the access point, $R S S_{A P_{i}, p}$ represents the average signal strength received at the sampling point position $p$ from No. $I$ access point. The basic parameters are shown in Table 1:

\section{Table 1. Parameters Values}

\begin{tabular}{l|l}
\hline Parameter & Value \\
\hline$P l\left(d_{0}\right)$ & $41.5 \mathrm{dBm}$ \\
$\alpha$ & 2 \\
$P t$ & $15 \mathrm{dBm}$ \\
\hline
\end{tabular}

In order to imitate the real environment, in experiments, the average signal strength received at each test point was added with Gauss noise with the mean $\mu=0$ and variance $\sigma=1$.

\subsubsection{Experimental Results}

As can be seen from Figure 2, SVR algorithm RMSE is significantly better than KNN algorithm, SK algorithm RMSE outweighs SVR, and with the increasing of the number of test points, KNN algorithm RMSE stabilizes at around $1.59 \mathrm{~m}$, SVR algorithm RMSE stabilizes at around $1.42 \mathrm{~m}$, SK algorithm RMSE stabilizes at about $1.26 \mathrm{~m}$, respectively lower than SVR and KNN algorithm RMSE by about $10 \%$ and $17 \%$. 


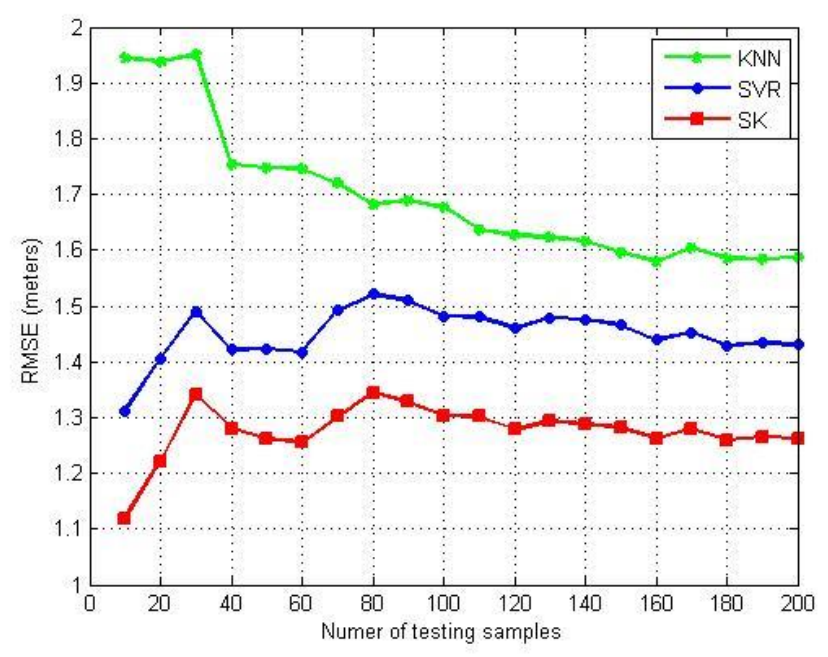

Figure 2. Comparison of RMSE

Figure 3 shows in terms of $2 \mathrm{~m}$ positioning error confidence probability, SVR positioning algorithm outweighs KNN in properties, SK positioning outweighs SVR in properties. In case of 200 test points, KNN confidence probability was 78\%, SVR confidence probability was $88 \%$. The latter outweighed the former by $10 \%$. SK confidence probability was $94 \%$, outweighing SVR by $6 \%$. Overall, with the increase in the number of test points, each algorithm confidence probability is stable.

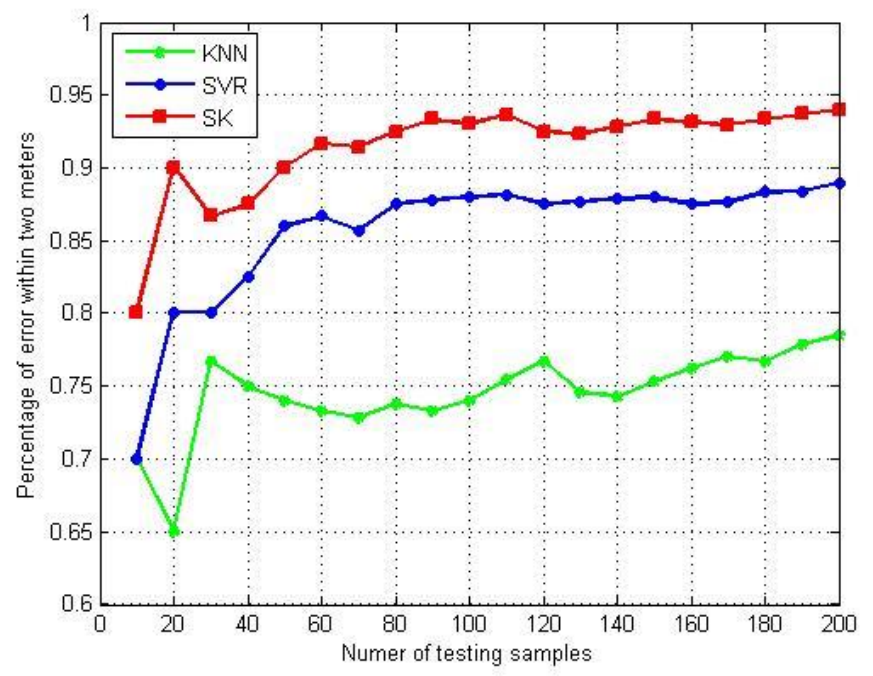

Figure 3. Comparison of Percentage of Error within 2 Meters

As can be seen from Figure 4, in case of the error distance within 3 meter, SK outweighs KNN and SVR in confidence probability. In addition, in case the positioning error with a confidence probability within $80 \%$ is considered as the indicator of positioning accuracy, SK algorithm can improve the positioning accuracy within $80 \%$ from KNN algorithm 2 meters to 1.5 meters, improving positioning properties by $25 \%$. 


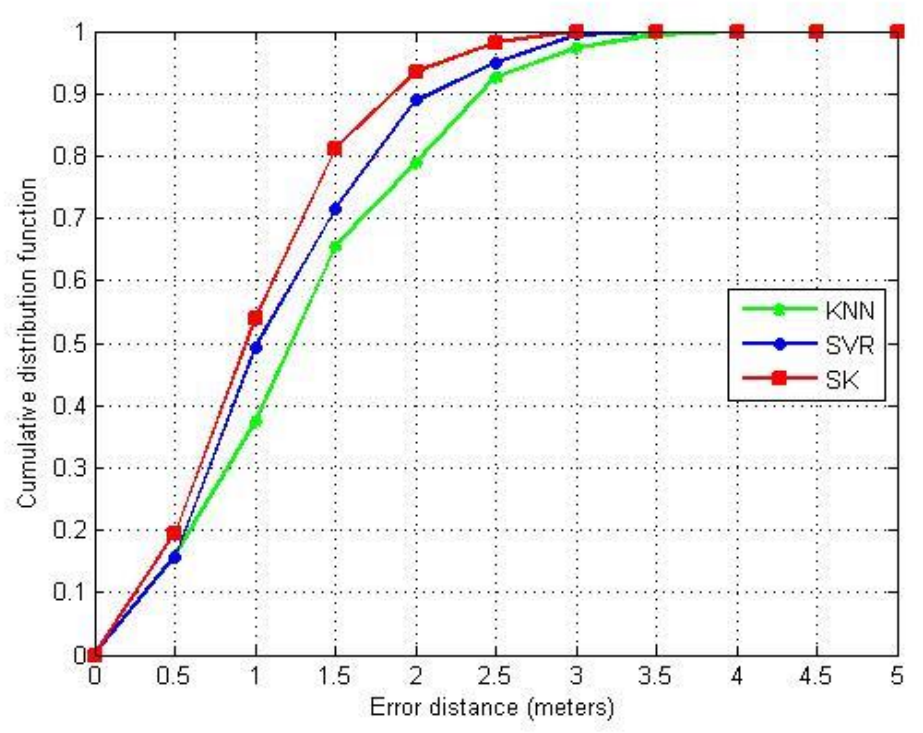

Figure 4. Comparison of CDF

\subsection{Realistic Scene}

\subsubsection{Establishment of the Realistic Scene}

This paper's indoor positioning experimental environment is Floor 4 of a lab building, with a length of 50 meter, a width of 20 meters, and 13 AP, a distance between AP of about 20 meters. Test area includes the corridor and halls on its sides. Experimental site sampling path is shown in Figure 5. The dots in the figure represent reference points, squares represent test points, and arrows represent the path and direction.

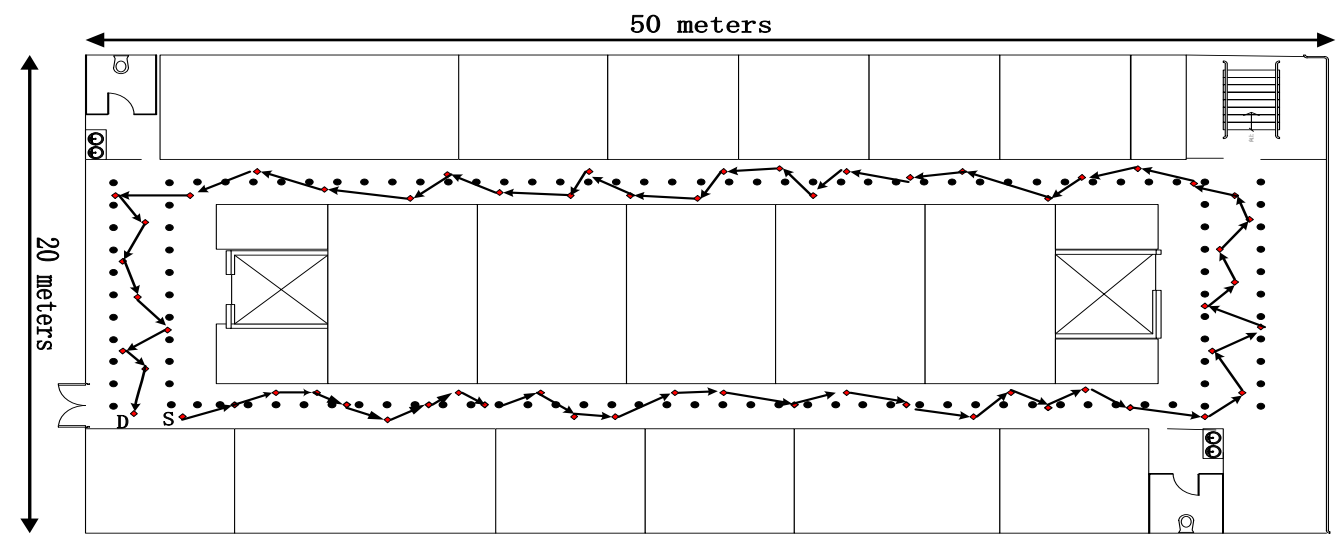

Figure 5. Realistic Scene

In the offline phase, 116 reference points were adopted, the spacing between the reference points was 1 meter, the sampling time of each reference point was 180 seconds, that is, each reference point reading signal strength was 180 times (terminal signal strength scanning frequency was once/s). In the online real-time follow-up phase, 60 test points were adopted, each test point sampling time was 30 seconds, that is, each test point reading signal strength was 30 times. 


\subsubsection{Experimental Results}

Figure 6 shows that with the increase in the number of test points, each algorithm RMSE stabilized, SK outweighed KNN and SVR algorithm in properties, SVR slightly outweighed KNN in properties. In case of 60 test points, SK was lower than KNN and SVR in RMSE by $28 \%$ and $16 \%$ respectively.

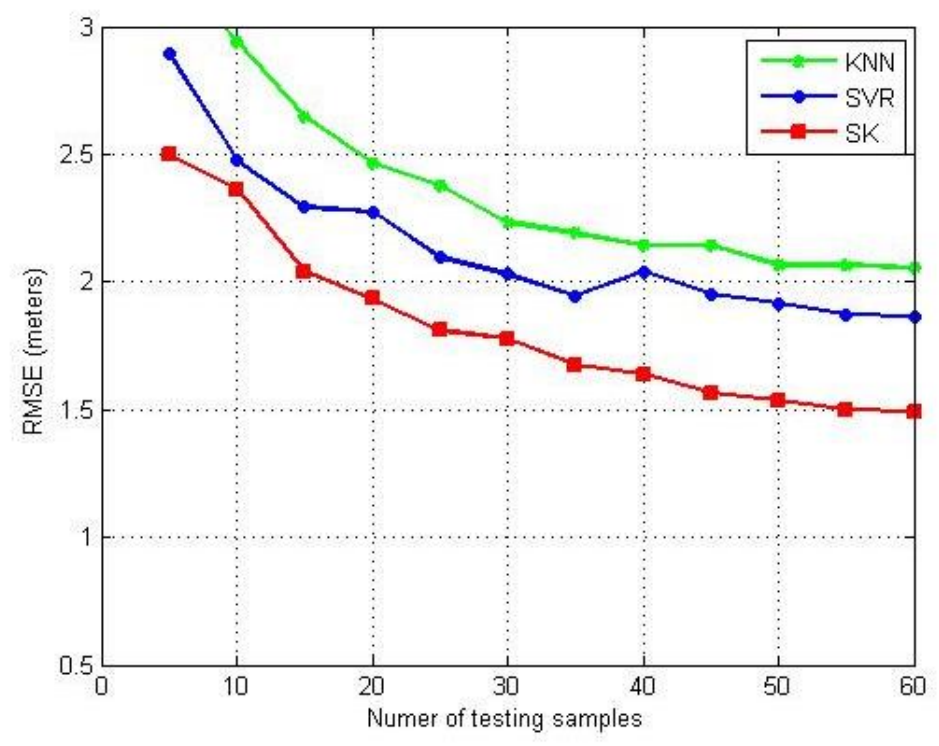

Figure 6. Comparison of RMSE

Figure 7 shows the changes in positioning error confidence probability of each algorithm within 2 meters with increasing of the number of test points. SVR algorithm evidently outweighed KNN in confidence probability. SK algorithm significantly outweighed SVR algorithm in confidence probability. In case of 60 test points, SK outweighed $\mathrm{KNN}$ in confidence probability by $24 \%$. In terms of environment simulation results, the overall confidence probability decreased, indicating the positioning results with the positioning error greater than 2 meters increased with the increase of the number of test points against the realistic environment.

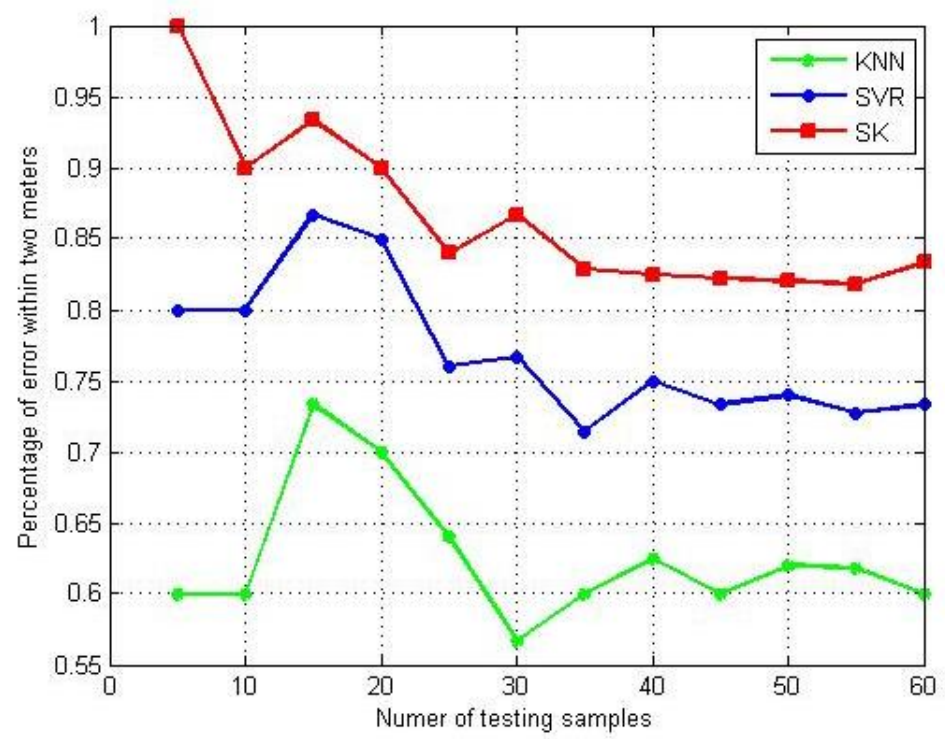

Figure 7. Comparison of Percentage of Error within 2 Meters 
Figure 8 shows the confidence probability of each algorithm against different positioning error distances. SK outweighed the other two algorithms. SVR overall outweighed KNN in properties slightly, especially in case the positioning error was greater than 2 meters. Compared with Figure 4, the properties of all algorithms were the same.

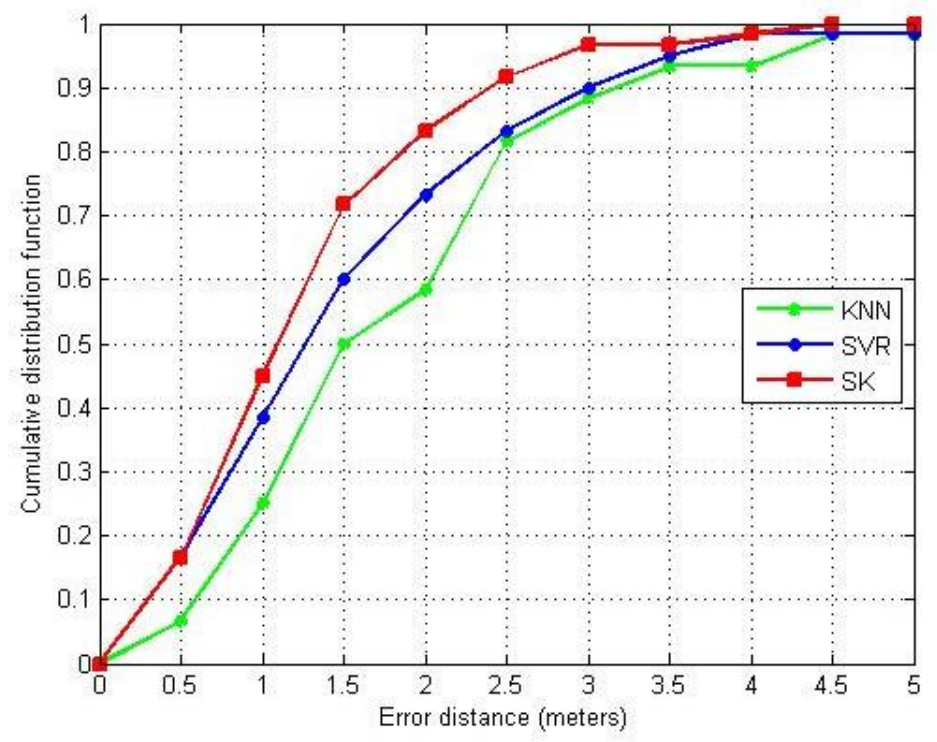

Figure 8. Comparison of CDF

Figure 9 shows changes in X coordinate after SVR positioning results were processed with Kalman Filtering. As can be seen from the figure, filtering can significantly correct SVR positioning results, making it imitate the real X coordinate, reducing the error, which shows Kalman algorithm can balance the positioning results, making the user's position mobile regular.

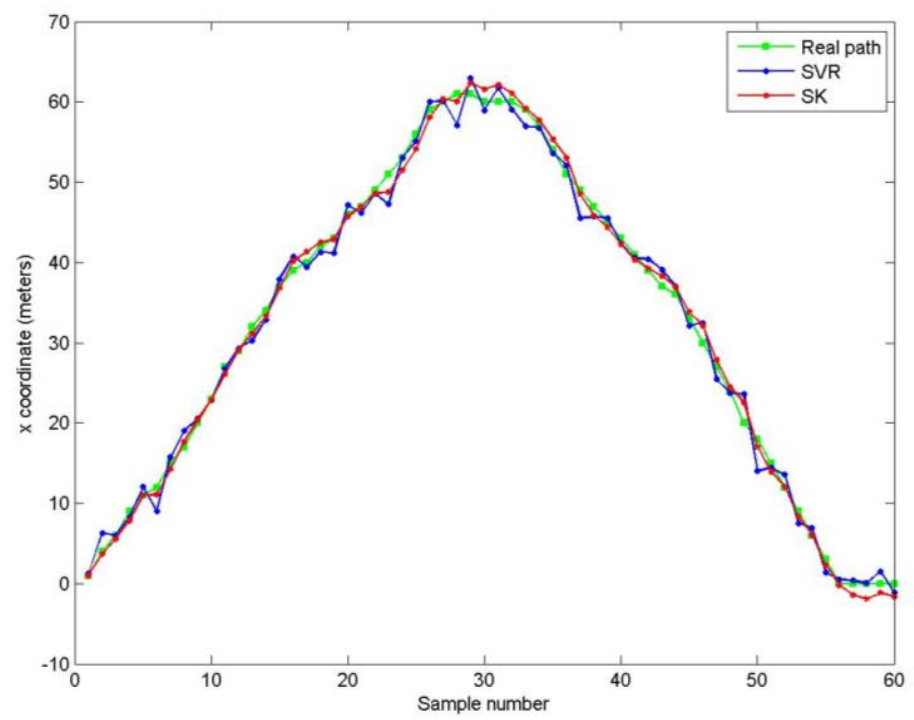

Figure 9. Kalman Filtering Simulator of $x$ Coordinate 


\section{Conclusion and Future Work}

This paper adopted SVR to achieve the indoor positioning, conducted training with the data obtained through signal filtering in the offline phase so as to obtain the position prediction model, which was utilized to estimate the position coordinates of the moving target in the online phase. In order to make the user's position regular, Kalman Filtering was taken to filter and correct SVR positioning results, and the properties of the algorithm was verified against the simulation environment and realistic environment. Experimental results show that the positioning accuracy and stability of the system were improved. This experiment was characterized by fewer reference points and test points of the realistic scene, and narrow sampling space, which affected the properties of the SVR algorithm to a certain extent. It is planned to extend this experiment in the future, collecting more test points in multiple subspaces. It is also planned to try to utilize the extended Kalman Filtering and particle filtering algorithm to process the estimated user's position so as to verify whether the system's positioning properties are improved.

\section{Acknowledgements}

This work is support by Shanghai Municipal Bureau of Quality and Technology Supervision of China (no. 2014QK140), the National Natural Science Foundation of China (no. 61372086), and the Foundation of Science and Technology Commission of Shanghai (no. 13511500300).

\section{References}

[1] M. Ficco, R. Piatrantuono and S. Russo, "Supporting Ubiquitous Location Informationin Interworking 3G and Wireless Networks", Communications on ACM, vol. 53, no. 11, (2010), pp. 116-123.

[2] H. Liu, H. Darabi, P. Banerjee and J. Liu, "Survey of wireless indoor positioning techniques and systems", IEEE Trans. System, vol. 37, no. 6, (2007), pp. 1067-1080.

[3] B. Igor, C. Matteo and L. Fabio, "A Trainingless WiFi Fingerprint Positioning Approach Over Mobile Devices", IEEE Antennas and Wireless Propagation Letters, no. 13, (2014), pp. 832-835

[4] A. Krishnakumar and P. Krishnan, "On the Accuracy of Signal Strength-Based Estimation Techniques", Proc. IEEE INFOCOM, no. 1, (2005), pp. 642-650.

[5] X. Chai and Q. Yang, "Reducing the Calibration Effort for Probabilistic Indoor Location Estimation", IEEE Trans. Mobile Comput., vol. 6, no. 6, (2007), pp. 649-662

[6] S.P. Kuo and Y C. Tseng, "Discriminant minimization search for largescale RF-based localization systems", IEEE Trans, Mobile Computer, vol. 10, no. 2, (2011), pp. 291-304.

[7] D. Redzic, B. Conor and E. O'Cnnor, "SEAMLOC:Seamless Indoor Localization Based on Reduced Number of Calibration Points", IEEE Transactions on Mobile Computer, vol. 13, no. 6, (2014), pp. 1326-1337.

[8] S.H. Fang and T.N. Lin, "Principal Component Localization in Indoor WLAN Environments", IEEE Transactions on Mobile Computer, vol. 11, no. 1, (2012), pp. 100-110.

[9] B. Wang, S.L. Zhou, W.Y. Liu and Y.J. Mo, "Indoor Localization Based on Curve Fitting and Location Search Using Received Signal Strength”, IEEE Transactions Industrial Electronics, vol. 62, no. 1, (2015), pp. $572-582$.

[10] K. Kaemarungsi and P. Krishnamurthy, "Modeling of Indoor Positioning Systems Based on ocation Fingerprinting", Proceeding of INFOCOM, (2004), Hong Kong, China.

[11] X. Li and K. Pahlavan, "Super-Resolution TOA Estimation with Diversity for Indoor Geolocation", IEEE Trans.Wireless Comm., vol. 3, no. 1, (2004), pp. 224-234.

[12] D. Niculescu and B. Nath, "VOR Base Stations for Indoor 802.11 Positioning", Proceeding of Mobile Computer, (2004), Philadelphia, USA.

[13] M. Brunato and R. Battiti, "Statistical Learning Theory for Location Fingerprinting in Wireless Lan", s. Computer Networks, vol. 47, no. 6, (2005), pp. 825-845.

[14] Z.L. Wu, C.H. Li, J.Y. Ng and K. Leung, "Location Estimation via Support Vector Regression", IEEE Transactions on Mobile Computer, vol. 6, no. 3, (2007), pp. 311-321.

[15] M. Youssef and A. Agrawala, "Handling Samples Correlation in the Horus System", Proceeding of INFOCOM, (2004), Hong Kong, China.

[16] P. Bahl and V. Padmanabhan, "RADAR: An in-building RF-based User Location and Tracking System", Proceeding of INFOCOM, (2000), Tel Aviv, Israel.

[17] M. Youssef and A. Agrawala, "The HORUS location determination system", Wireless Networking, vol. 14, no. 3, (2008), pp. 357-374. 
[18] Ekahau Positioning Engine 4, vol. 1 [OL], http://www.ekahau.com

[19] J.J. Pan, S.J. Pan, J. Yin, "Tracking Mobile Users in Wireless Network via Semi-Supervised Colocalization”, IEEE Transactions on Pattern Analysis and Machine Intelligence, vol. 34, no. 3, (2012), pp. 587-600.

[20] T.S. Rappaport, "Wireless Communications: Principles and Practice", Upper Saddle River, New Jersey, USA: Prentice Hall PTR, (1996).

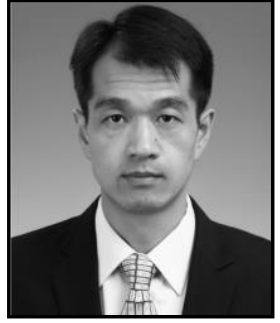

Yu Zhang, He received MS degree in computer engineering from Harbin university of Science and Technology in 2006, and the $\mathrm{PhD}$ degree from East China Normal University in 2013. He is currently postdoctoral researcher working on Shanghai Institute of Measurement and Testing Technology of China. His currently research interests include indoor localization, resource management and optimization of wireless communication networks.

Lian Dong, She received BS degree in physics from Tsinghua

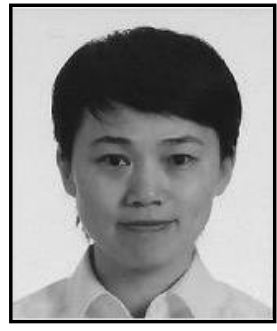
University in 1988, now she is a professor in Shanghai Institute of Measurement and Testing Technology of China, and as a leader of navigation team. She research interests include measurement technology of satellite navigation products and indoor localization.

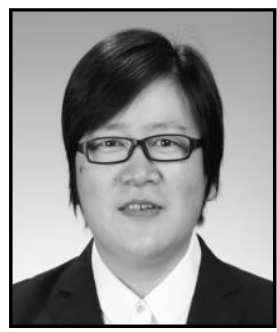

Lei Lai, She received MS degree from DongHua University, China, in 2008. Now she is a senior engineer in Shanghai Institute of Measurement and Testing Technology of China. She research interests include measurement technology of satellite navigation products and indoor localization.

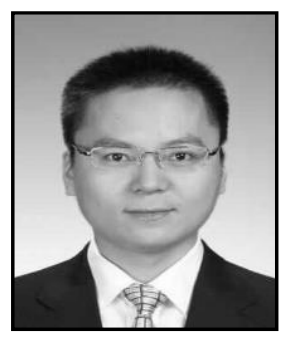

Lizhi Hu, He received bachelor's degree and MS degree from National Institute of Metrology, China, in 2006 and 2009, respectively. Now $\mathrm{He}$ is an engineer in Shanghai Institute of Measurement and Testing Technology of China. He research interests include measurement technology of satellite navigation products and indoor localization. 
International Journal of Future Generation Communication and Networking Vol. 9, No. 3 (2016) 